\title{
On a labeled vehicule routing problem
}

\section{Hatem Chatti, Laurent Gourvès, Jérôme Monnot}

CNRS - Université Paris Dauphine

January 26, 2010 


\section{Outline}

(1) motivation of labeled optimization

(2) definition of the labeled VRP

(3) hardness

(3) approximation

(3) concluding remarks 


\section{Introduction}

Typical combinatorial optimization problems

Given a set of weighted facilities (e.g. edges or vertices of a graph), find a feasible subset of facilities with optimum (i.e. minimum or maximum) total weight

e.g. minimum weight spanning tree, TSP, etc

\section{Labeled optimization problems}

Given a set of labeled facilities, find a feasible subset of facilities with optimum number of labels assumption: either all facilities having the same label are purchased or none of them 


\section{Motivation}

Weights often represent a cost/distance/duration while a label (or color) can represent

- a mode of transport (plane, bus, metro, etc)

- a technology (optical fiber, encryption)

- name of the facility's owner (a company)

Sometimes the quality of a solution is not dominated by its total distance

- number of changings in the metro

- direct flights are sometimes more expensive than trips with one stop in the opposite direction 


\section{The vehicle routing problem (VRP)}

\section{Classical VRP}

A complete graph with

- a depot denoted by 0

- $n$ customers denoted by $1, \ldots, n$

- a capacity $k$

- a distance on the edges

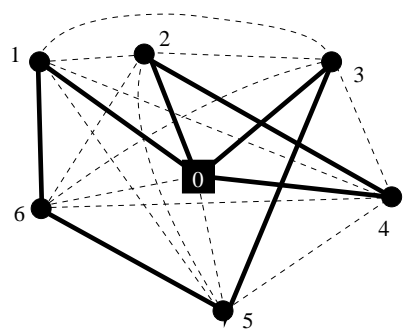

Find a collection of cycles such that

- every vertex/customer is visited exactly once

- every cycle contains the depot plus at most $k$ customers

- the total distance is minimized 


\section{The LABELED vehicle routing problem (LVRP)}

\section{VRP with labels}

A complete graph with

- a depot denoted by 0

- $n$ customers denoted by $1, \ldots, n$

- a capacity $k$

- a color on each edge color=label

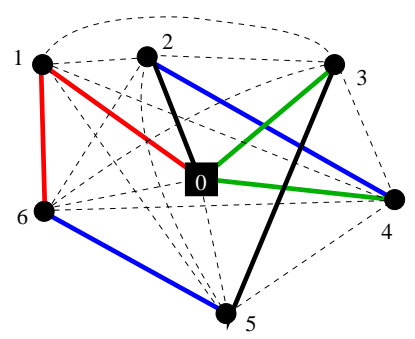

Find a collection of cycles such that

- every vertex/customer is visited exactly once

- every cycle contains the depot plus at most $k$ customers

- the number of colors used is minimized 


\section{Approach}

- identify polynomial/NP-hard cases of the problem

- poly-time approximation algorithms for NP-hard cases

- approximation ratio: $\frac{S O L}{O P T}$ in the worst case

\section{bad news}

Unless $P=N P$, and taking $\epsilon \in(0,1)$, there is no $n^{1-\epsilon}$-approximation algorithm for $\operatorname{LVRP}(k)$, where $k \geq n^{\epsilon}$

A trivial algorithm gives an $(n+1)$-approximation 


\section{frequencies}

The frequency of a color is the number of times it appears in the graph

$f$ denotes the maximum frequency in the graph

For a set of edges $E^{\prime}, f\left(E^{\prime}\right)$ is the maximum frequency for the colors appearing in $E^{\prime}$ 


\section{Simple observations}

\section{observation 1}

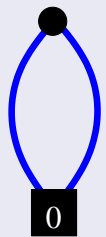

one can visit a customer using a color and return back to the depot at "no cost"

\section{observation 2}
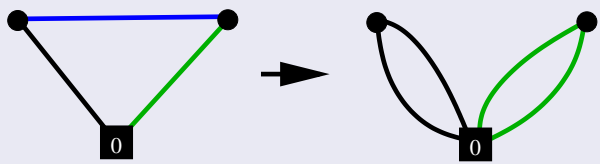

every cycle of length 3 can be replaced by two cycles of length one (the number of colors cannot increase) assumption: no cycle of length 3 


\section{Simple observations}

\section{observation 3}

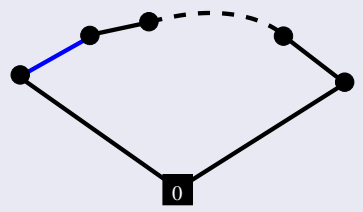

The blue color must appear twice. Otherwise, replace by:

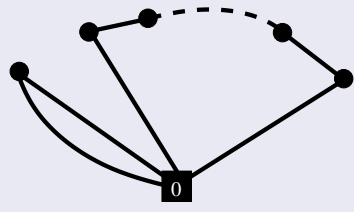




\section{NP-hardness}

LVRP is polynomial if $k=1,2$ or $f=1$. What about the case $k=3$ and $f=2$ ?

$k$-partition problem

decide whether a simple graph on $k q$ vertices can be partitionned into $q$ pathes of length $k-1$
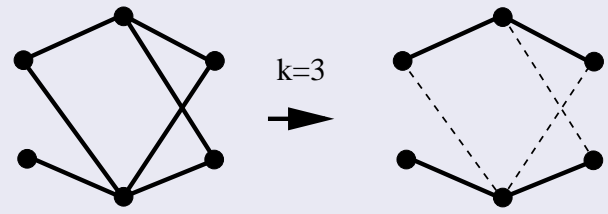

NP-complete problem when $k \geq 3$ 


\section{NP-hardness of LVRP when $k=3$ and $f=2$}

\section{reduction of the 3-partition problem}

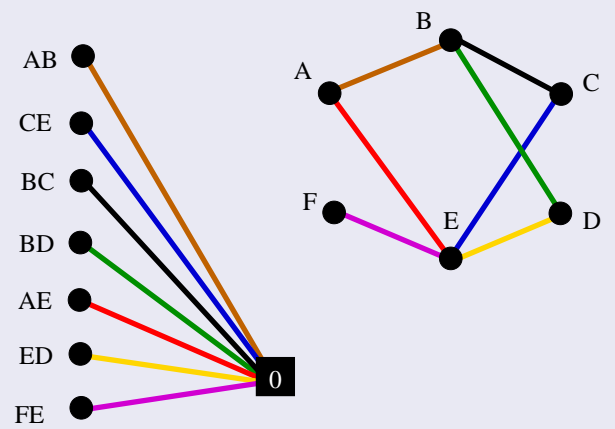

- keep all vertices of $G$, add a depot 0 and add one "edge-vertex" $v_{e}$ per edge $e$ of $G$

- one color per edge $e$ in $G$ and edge $\left(0, v_{e}\right)$

- any other edge receives a unique color 


\section{NP-hardness of LVRP when $k=3$ and $f=2$}

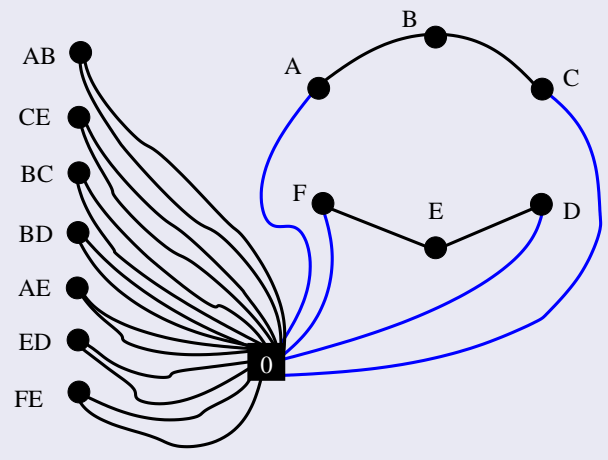

$G$ admits a 3-partition iff there is a solution using less than $m+2 q$ colors

- every "edge-vertex" is covered by a cycle of length 2

- the other vertices are in cycles of length $k+1$ 


\section{Approximation}

We suppose that we cover every customer with a cycle of length 2, i.e. the approximate solution is a star with the depot at the center

\section{Notations}

$E_{0}=\{[0, i]: 1 \leq i \leq n\}$ is the star (approximate solution)

$E^{*}$ is the optimal solution

$s_{i}$ is the number of cycles of length $i$ in the star

$s_{i}^{*}$ is the number of cycles of length $i$ in the optimal solution

\section{Two cases}

- $f \leq \frac{k+1}{2}$

approx. ratio $\frac{f(k-2)+1}{k-1}$

presented

- $f \geq \frac{k+1}{2}$

approx. ratio $\frac{f(k-2)+k+1}{k+1}$

skipped 


\section{Case $f \leq(k+1) / 2$}

For a set of edges $E^{\prime}, R\left(E^{\prime}\right)$ is defined as $\left|E^{\prime} \cap E_{0}\right|$ minus the number of colors in $E^{\prime} \cap E_{0}$ (colors saved for the edges adjacent to the depot)

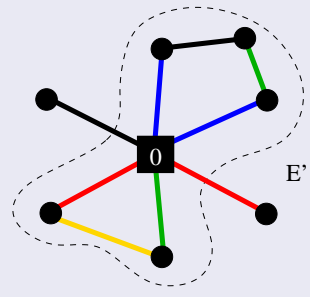

$R\left(E^{\prime}\right)=4-3=1$ in the example

$$
O P T \geq s_{2}^{*}+2 \sum_{i=4}^{k+1} s_{i}^{*}-R\left(E^{*}\right)
$$




\section{$O P T \geq s_{2}^{*}+2 \sum_{i=4}^{k+1} s_{i}^{*}-R\left(E^{*}\right)$}




\section{$O P T \geq s_{2}^{*}+2 \sum_{i=4}^{k+1} s_{i}^{*}-R\left(E^{*}\right)$}

$$
O P T \geq\left(s_{2}^{*}+\sum_{i=4}^{k+1} i s_{i}^{*}\right) / f
$$

$(\alpha+\beta) O P T \geq\left(\frac{\alpha}{f}+\beta\right) s_{2}^{*}+\sum_{i=4}^{k+1}\left(\frac{\alpha}{f}+2 \beta\right) s_{i}^{*}-\beta R\left(E^{*}\right)$

Take $\alpha=f \frac{k-2}{k-1}$ and $\beta=\frac{1}{k-1}$

We have $\frac{\alpha}{f}+\beta \geq 1, \frac{\alpha}{f}+2 \beta \geq i-1$ and $\beta \leq 1$

$$
\begin{aligned}
\frac{f(k-2)+1}{k-1} O P T & \geq s_{2}^{*}+\sum_{i=4}^{k+1}(i-1) s_{i}^{*}-R\left(E^{*}\right) \\
& \geq n-R\left(E^{*}\right) \geq S O L
\end{aligned}
$$




\section{Tightness}

One can show examples where the star is $\frac{f(k-2)+1}{k-1}$-approximate for every $k$ and $f$ satisfying $f \leq \frac{k+1}{2}$

$$
k=4 \text { and } f=2
$$

instance

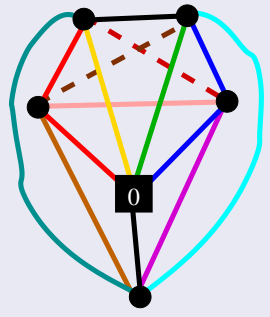

optimum $=3$

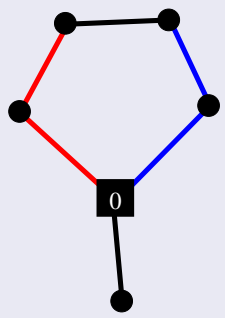

sol $=5$

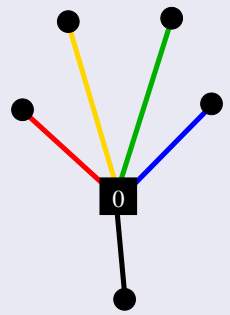




\section{Other results}

LVRP when $k=3,4, f=2$ is APX-hard

LVRP when $k=3, f=2$ is polynomial if every color of the star has frequency 1

LVRP when $k=3, f=3$ is NP-hard even if every color of the star has frequency 1

With local improvements performed on the star, one can improve the previous approximation ratio if $R\left(E_{0}\right)=0$ 
Thank You 